ARTICLE

\title{
MicroRNA 139-5p coordinates APLNR-CXCR4 crosstalk during vascular maturation
}

Irinna Papangeli ${ }^{1, \star}$, Jongmin Kim ${ }^{1,2, \star}$, Inna Maier ${ }^{3}$, Saejeong Park ${ }^{1}$, Aram Lee ${ }^{2}$, Yujung Kang ${ }^{1}$, Keiichiro Tanaka ${ }^{1}$, Omar F. Khan ${ }^{4}$, Hyekyung Ju${ }^{1}$, Yoko Kojima', Kristy Red-Horse ${ }^{5}$, Daniel G. Anderson ${ }^{4}$, Arndt F. Siekmann ${ }^{3} \&$ Hyung J. Chun ${ }^{1}$

G protein-coupled receptor (GPCR) signalling, including that involving apelin (APLN) and its receptor APLNR, is known to be important in vascular development. How this ligand-receptor pair regulates the downstream signalling cascades in this context remains poorly understood. Here, we show that mice with Apln, Aplnr or endothelial-specific Aplnr deletion develop profound retinal vascular defects, which are at least in part due to dysregulated increase in endothelial CXCR4 expression. Endothelial CXCR4 is negatively regulated by miR-139-5p, whose transcription is in turn induced by laminar flow and APLN/APLNR signalling. Inhibition of miR-139-5p in vivo partially phenocopies the retinal vascular defects of APLN/APLNR deficiency. Pharmacological inhibition of CXCR4 signalling or augmentation of the miR-139-5p-CXCR4 axis can ameliorate the vascular phenotype of APLN/APLNR deficient state. Overall, we identify an important microRNA-mediated GPCR crosstalk, which plays a key role in vascular development.

\footnotetext{
${ }^{1}$ Department of Internal Medicine, Yale Cardiovascular Research Center, Section of Cardiovascular Medicine, Yale University School of Medicine, 300 George Street, 7th Floor, New Haven, Connecticut 06511, USA. ${ }^{2}$ Department of Life Systems, Sookmyung Women's University, Seoul 140-742, Korea. ${ }^{3}$ Max Planck Institute for Molecular Biomedicine, Roentgenstr. 20, 48149 Muenster, Germany. ${ }^{4}$ David H. Koch Institute for Integrative Cancer Research, Massachusetts Institute of Technology, Cambridge, Massachusetts 02142, USA. ${ }^{5}$ Department of Biological Sciences, Stanford University, Stanford, California 94305 , USA.

* These authors contributed equally to this work. Correspondence and requests for materials should be addressed to H.J.C. (email: hyung.chun@yale.edu).
} 
A functional vascular system is fundamental for normal vertebrate development, as it is critical for delivery of oxygen and nutrients, while removing waste to ensure growth and differentiation of all tissues. The concept of tip and stalk cells has emerged over the past decade in the context of sprouting angiogenesis, and guides our understanding of the transition from vascular sprouting to maturation and ultimately quiescence $^{1,2}$. In this context, endothelial cell (EC) subsets can respond to molecular and biomechanical cues, and acquire non-proliferative tip cell identities which can extend numerous filopodia and guide vascular outgrowth, or become stalk cells that are considered proliferative and capable of forming lumens ${ }^{3}$. Both the vascular endothelial growth factor (VEGF) and the Notch signalling pathways have been extensively described to regulate the discrimination between these cell populations during mouse and zebrafish development ${ }^{3-8}$. Despite the important role and extensive characterization of the VEGF-Notch signalling paradigm, several additional tip and stalk cell-specific genes have been identified, including APLNR (stalk cells) and CXCR4 (tip cells), whose genetic deletions lead to profound vascular defects ${ }^{9-13}$. Moreover, several $G$ protein-coupled receptor (GPCR)-ligand combinations, including stromal cellderived factor 1 (SDF-1)/CXCR4, S1P/S1PR1 and apelin (APLN)/ APLNR, have been shown to play critical roles in vascular development ${ }^{9-12,14-16}$. Nevertheless, unresolved questions remain, and our understanding of the mechanisms by which these signalling processes are integrated remains incomplete.

Here we define a novel mechanism by which signalling pathways involving two of these GPCRs, namely APLNR and CXCR4, are intricately linked in the developing vasculature. We identify robust flow-induced endothelial expression of APLNR in both in vitro and in vivo models, which in turn leads to marked suppression of endothelial CXCR4 expression. This crosstalk was found to involve a key shear responsive microRNA (miRNA), miR-139-5p, which is positively regulated by APLN/APLNR signalling and directly targets CXCR4 in ECs. Moreover, we demonstrate novel pharmacological strategies to restore this signalling axis that rescue the vascular phenotypes associated with APLN/APLNR deficiency. Overall, these findings provide key mechanistic insights that involve a critical miRNA-based crosstalk between two GPCR signalling cascades, which regulate important early steps in vascular maturation.

\section{Results}

APLN/APLNR downregulates CXCR4 in developing retinal vessels. Our previous investigation of the APLN/APLNR signalling pathway included a gene expression profiling analysis of transcripts that are differentially regulated by APLN/APLNR knockdown in ECs (ref. 11). Of the transcripts that were increased, we found that CXCR4 was one of the most significantly upregulated transcripts in this context (data set GSE46825, Gene Expression Omnibus database (GEO NCBI)). We confirmed that both the messenger RNA (mRNA) and protein levels of CXCR4 are significantly increased in human umbilical vein ECs (HUVECs) subjected to APLN or APLNR knockdown (Fig. 1a-d). Conversely, flow cytometry analyses demonstrated significant decrease in CXCR4 expression in response to APLNR overexpression in HUVECs (Fig. 1e). To further validate this inverse correlation between APLNR and CXCR4 in vivo, we examined mice with genetic deletion of either Aplnr or Apln. Expression of Cxcr4 in postnatal day 5 (P5) retinas of both the Aplnr ${ }^{-/-}$and Apln ${ }^{-/-}$mice, as detected by in situ hybridization, was markedly increased compared with respective littermate controls (Fig. 1f). Moreover, to quantitatively assess the changes in endothelial Cxcr4 expression in vivo, we carried out flow cytometry analysis using retinas from $A p l n r^{-/-}$mice and found significantly higher expression of Cxcr4 in the retinal ECs compared with littermate controls (Supplementary Fig. 1). Both the $A p l n^{-1-}$ and Apln $r^{-/-}$mice also exhibited a significant delay in retinal vascular growth, decreased total vascularized area and vascular branchpoints at P5, as previously demonstrated (Supplementary Fig. 2a,b) ${ }^{12,17,18}$. To confirm that these phenotypes are specifically due to endothelial APLNR signalling, we investigated the retinal vascular phenotype in mice with conditional, endothelial-specific deletion of Aplnr (Aplnrfl/fl;Cdh5-CreERT2) using a tamoxifen inducible Cdh5 Cre driver (Cdh5-CreERT2) ${ }^{19}$ (Supplementary Fig. 3a,b). We found that mice lacking Aplnr selectively in the endothelium recapitulated the Aplnr global knockout phenotype in the retina, albeit less severely (Fig. 1g,h). These mice also exhibited higher levels of Cxcr4 expression in retinal ECs as analysed by flow cytometry (Supplementary Fig. 3c).

Prior studies have shown that loss of CXCR4 signalling results in spindle-like tip cells that fail to migrate properly ${ }^{10}$; however, the effects of excess CXCR4 are not known. To investigate whether the Apln ${ }^{-/-}$and Aplnr ${ }^{-/-}$vascular phenotypes are in part a consequence of increased CXCR4 signalling, we injected newborn mice with AMD3100, a known selective CXCR4 inhibitor $^{20}$. First, we confirmed that AMD3100 can inhibit effects of SDF- $1 \alpha$ in inducing endothelial sprouting in a fibrin gel bead assay (Supplementary Fig. 4). For our in vivo studies, as previous studies have demonstrated vascular abnormalities with high doses of AMD3100 (ref. 21), we chose a dose that was subphenotypic in control mice (Supplementary Fig. 5) and examined the retinal vasculature of the Aplnr ${ }^{-/}-$mice at P5. We found a significant increase in total vascularized area and vascular branchpoints of the Aplnr ${ }^{-/}$mice treated with AMD3100 compared with the PBS injected controls (Fig. 1i,j); the radial growth of the retinas was not significantly changed, suggesting a partial rescue of the $A p \ln r^{-/}$phenotype. Moreover, we also achieved partial rescue of the Aplnrfl/fl; Cdh5-CreERT2 mice with AMD3100, with increase in total vascularized area and trends towards increase in number of vascular branchpoints and radial growth (Supplementary Fig. 6a,b).

Endothelial APLNR expression is flow-dependent. We evaluated the retinal vasculature of P5 mice and confirmed that the expression of Aplnr was restricted to the stalk cells and maturing veins (Fig. 2a), whereas Apln was predominantly expressed by tip cell population (Fig. 2b), as previously reported ${ }^{12,22}$. We found that the expression of Aplnr was highest in the subset of ECs that surround the newly formed lumen, based on the perfusion pattern observed in fluorescein isothiocyanate (FITC) dextran injected P5 mice (Fig. 2c) ${ }^{3}$. Given the restricted Aplnr expression in the retinal vasculature to the leading edge of the lumenized network, which would be the endothelial subset exposed to early onset of blood flow, we evaluated whether shear stress may be a positive regulator of $A P L N R$ expression. The type of flow in this context has been modelled in silico ${ }^{23}$, but remains poorly characterized in vivo. We tested the effects of a number of different flow conditions on APLNR expression in HUVECs (Supplementary Fig. 7a). Of the different conditions of flow tested, we found the highest upregulation of APLNR with orbital shear stress (estimated to induce 10.7 dynes $\mathrm{cm}^{-2}$ ) 24 . We also conducted a serial time course to determine the effect of duration of flow on APLNR expression levels, and found that the highest induction was observed at $48 \mathrm{~h}$, while the expression levels began to decrease beyond that time (Supplementary Fig. 7b). Moreover, we found that upregulation of APLNR was at least in part dependent on the shear responsive transcription factors KLF2 
a

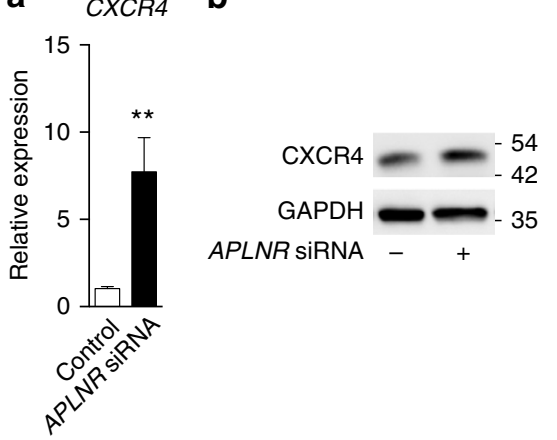

C

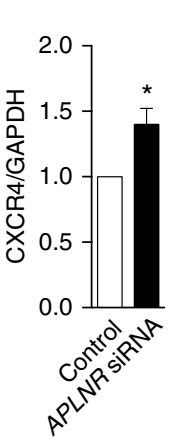

d

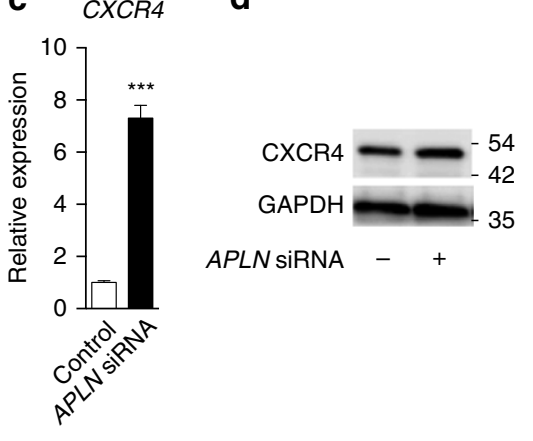

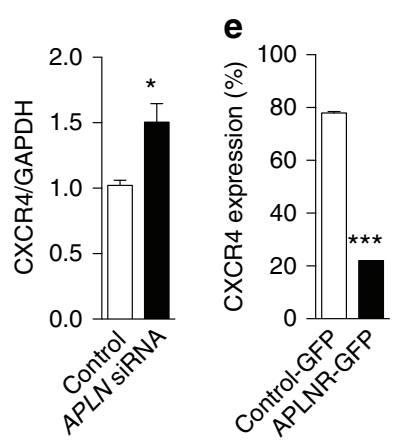

f
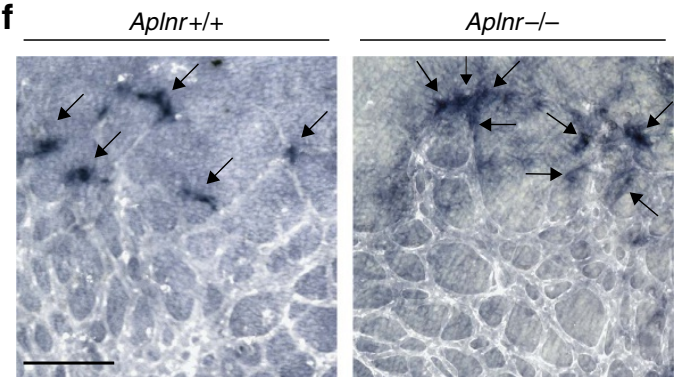

g
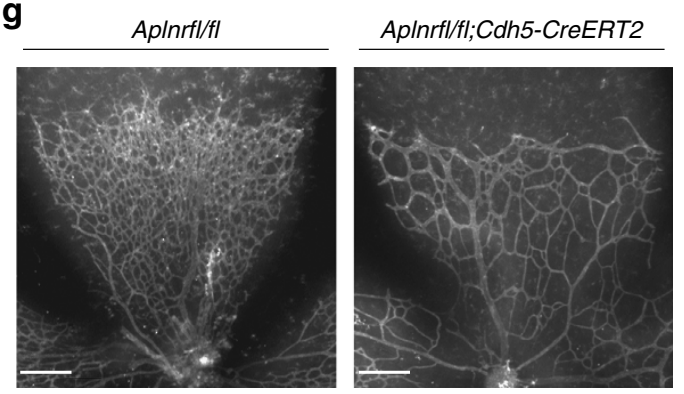

i

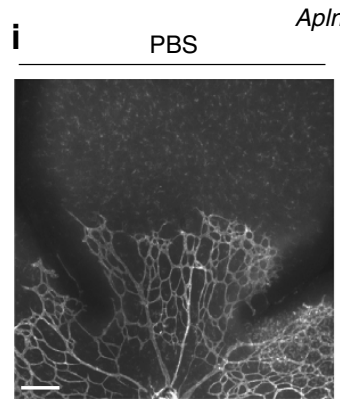

nr-l-

AMD3100

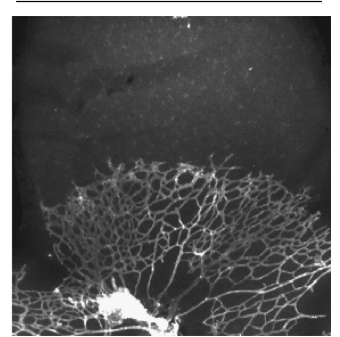

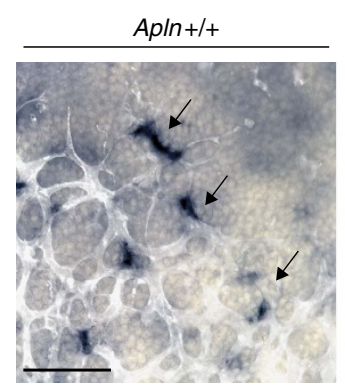

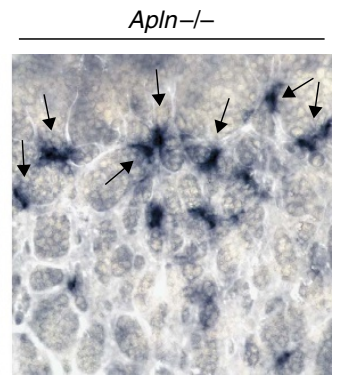

h
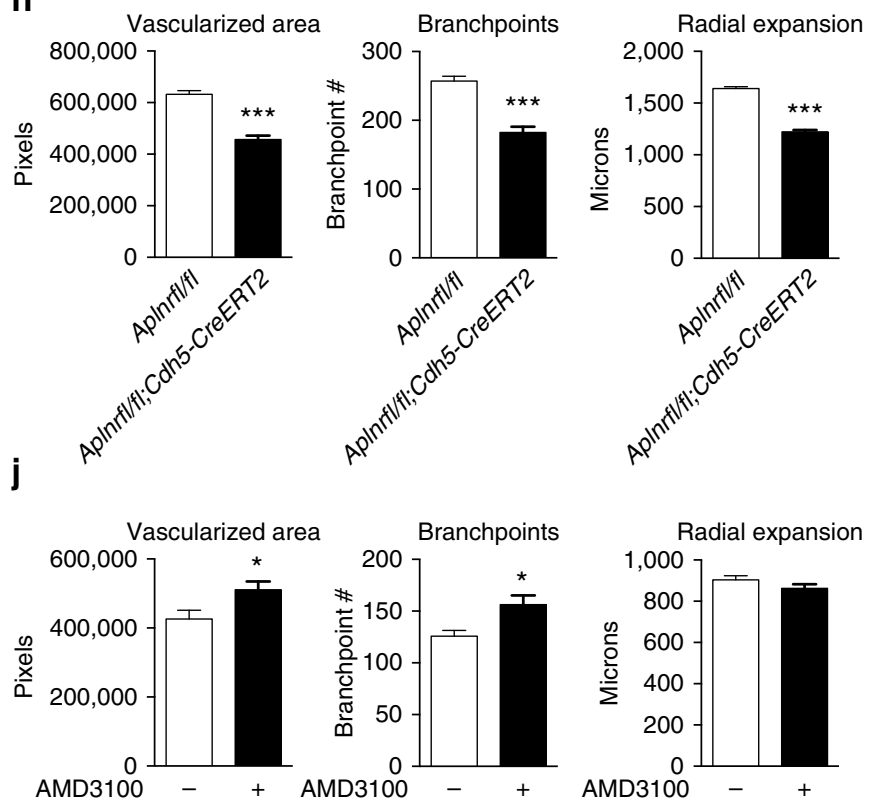

Figure 1 | Apelin/APLNR regulates CXCR4 expression in vitro and in vivo. (a-d) CXCR4 transcript and protein levels in response to siRNA-mediated knockdown of $\operatorname{APLNR}(\mathbf{a}, \mathbf{b})$ or $\operatorname{APLN}(\mathbf{c}, \mathbf{d}) . n=3$ experiments per condition. (e) Flow cytometry to determine level of CXCR4 expression in HUVECs transfected with either APLNR-GFP or GFP expression constructs. Data shown represents the CXCR4 staining of cells among the GFP-positive cells. $n=3$ experiments. (f) Cxcr4 expression (blue) in P5 retinal vessels as assessed by in situ hybridization in Aplnr ${ }^{-/-}$and Apln ${ }^{-/-}$mice. Arrows indicate Cxcr4 mRNA localization. Isolectin B4 is shown in white. Scale bar, $100 \mu \mathrm{m} . n=8$ retinas per genotype. (g,h) Retinal vasculature of P5 mice with endothelial-specific deletion of Aplnr (Ap/nrfl/fl;Cdh5-CreERT2). Graphs depict vascularized area, branchpoints and radial expansion. Scale bar, $200 \mu \mathrm{m}$. $n \geq 6$ retinas per genotype. (i,j) Effect of AMD3100 on $A p / n r^{-/-}$retinal vasculature. Scale bar, $200 \mu \mathrm{m} . n \geq 5$ retinas per genotype. ${ }^{\star} P<0.05,{ }^{\star \star} P \leq 0.01$, ${ }^{\star \star \star} P \leq 0.001$, $t$-test. Error bars represent s.e.m.

and KLF4, as knockdown of KLF2, KLF4 or both, markedly inhibited the shear-mediated APLNR upregulation (Fig. 2d). In addition, ERK5 knockdown, a known shear responsive MAP kinase upstream of KLF2 and KLF4 (ref. 25), also abrogated induction of APLNR expression under shear conditions (Fig. 2e). In contrast to APLNR, APLN expression was significantly downreguated after exposure of HUVECs to shear stress (Supplementary Fig. 8). Lastly, we investigated the role of the second APLNR ligand, namely Toddler (ELABELA) ${ }^{26,27}$ and found no effect on CXCR4 expression levels by either its endogenous knockdown or stimulation with exogenous peptide in HUVECs (Supplementary Fig. 9a,b). 
a

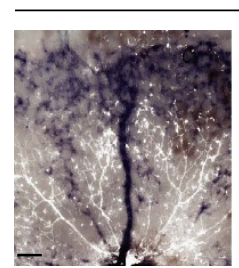

C

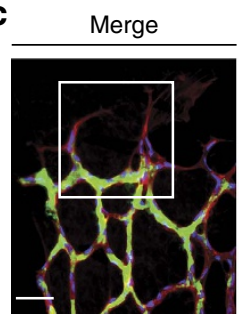

d

(2)

ApInr ISH

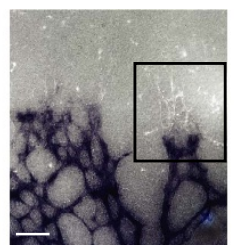

Merge
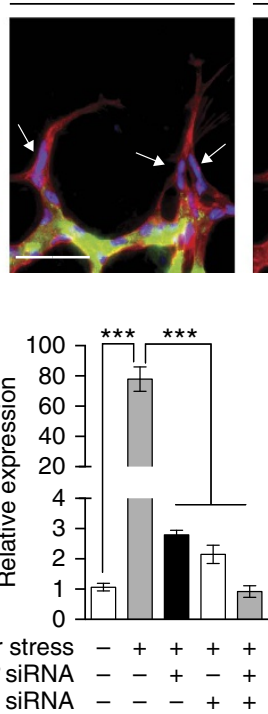

b

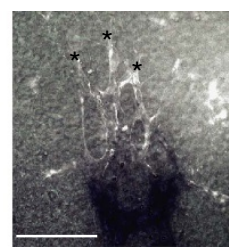

IsoB4

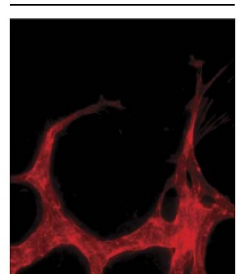

$\mathbf{f}$
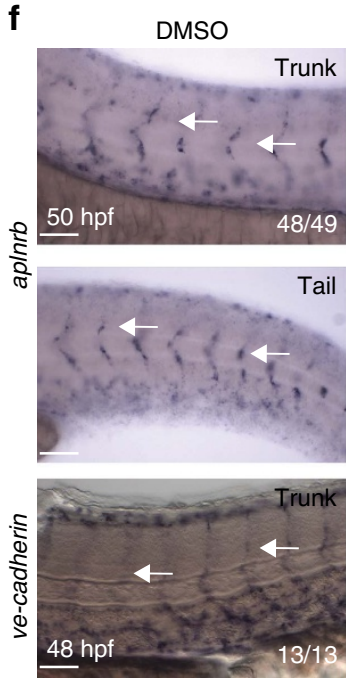
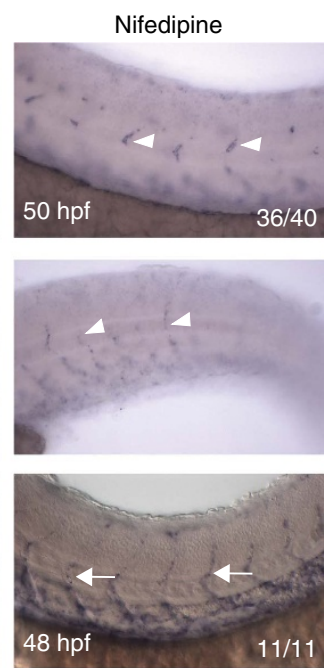

Apln ISH

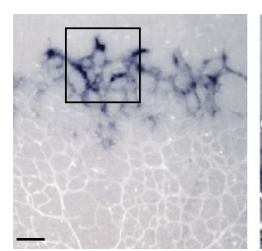

FITC Dextran

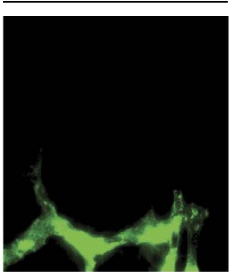

e

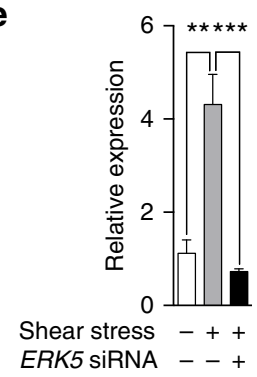

g
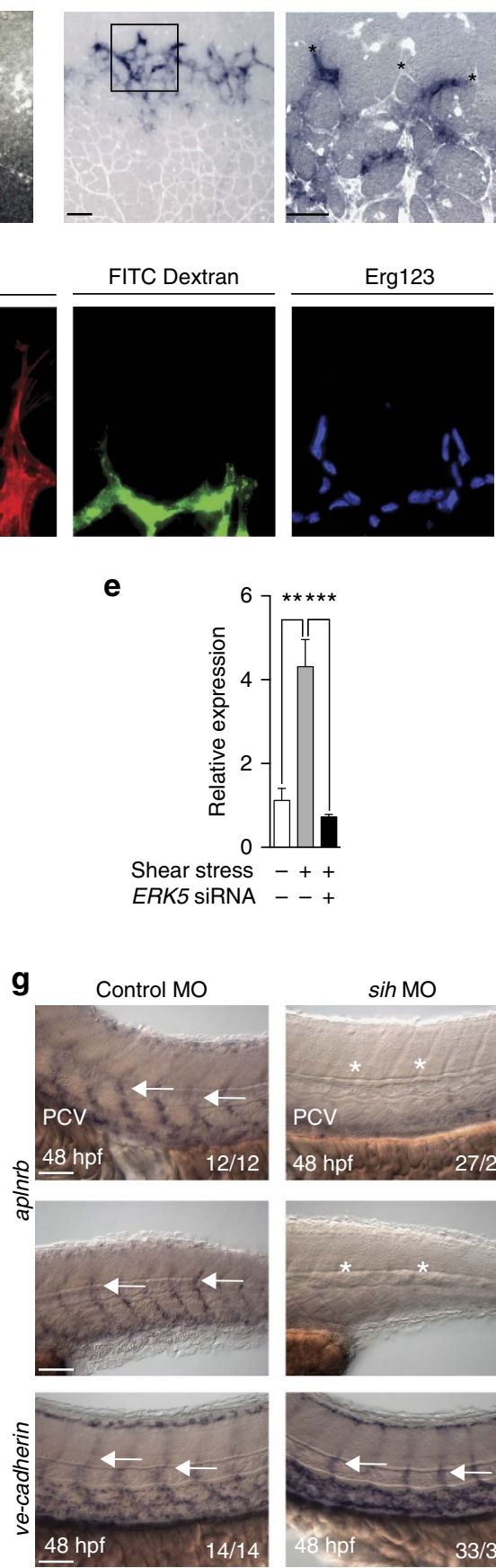
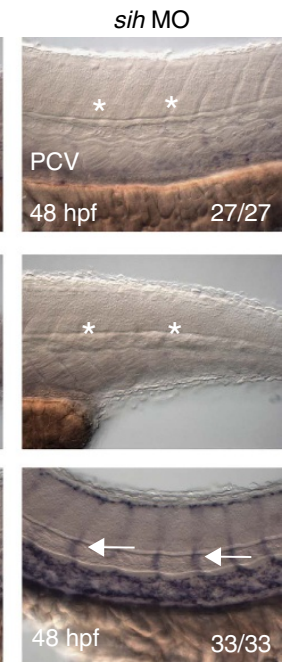

Figure 2 | APLNR is expressed in newly lumenized vessels and its expression is flow-dependent. (a) Aplnr expression as determined by in situ hybridization (blue) in P5 retinal vasculature with Isolectin B4 staining (white). The boxed area in the middle image is magnified on the right to show higher magnification of the adjacent panel. Scale bar, $50 \mu \mathrm{m} . n=3$ retinas. (b) Apln expression as determined by in situ hybridization (blue) in P5 retinal vasculature with Isolectin B4 staining (white). The boxed area is magnified on the right to show higher magnification of the adjacent panel. Scale bar, $100 \mu \mathrm{m}$ (low magnification) or $50 \mu \mathrm{m}$ (high magnification). $n=4$ retinas. Tip cells are marked by the asterisks. (c) Localization of FITC Dextran in the P5 retinal vasculature (green) with Isolectin B4 (red) and Erg123 (endothelial nuclei) staining (blue). The higher magnification images are shown in the right panels. Scale bar, $50 \mu \mathrm{m} . n=4$ retinas. (d) APLNR transcript expression in response to shear stress alone or with concurrent KLF2, KLF4 or combined knockdown via siRNA. (e) APLNR transcript expression in response to shear stress alone or with concurrent ERK5 knockdown via siRNA. (f) Aplnrb expression in the trunk and tail of zebrafish embryos at $50 \mathrm{hpf}$ with or without nifedipine ( $2 \mathrm{~h}$ treatment). Arrows (DMSO) and arrowheads (nifedipine) demarcate aplnrb expression. (g) Aplnrb expression in the trunk and tail of zebrafish embryos at $48 \mathrm{hpf}$ with sih or control morpholino (MO) injection. Asterisks mark absence of aplnrb staining in intersegmental vessels (ISVs). Staining in the posterior cardinal vein (PCV) is also reduced. In situ hybridization with the pan-endothelial marker vascular endothelial cadherin (ve-cadherin) shows normal vascular morphology in all conditions. Arrows mark intersegmental blood vessels. Scale bar, $50 \mu \mathrm{m} .{ }^{\star \star} P \leq 0.01,{ }^{\star \star \star} P \leq 0.001, t$-test. Error bars represent s.e.m. 
To further validate the shear-mediated APLNR regulation in vivo, we utilized the zebrafish embryonic system to evaluate the expression of the two paralogous apln receptors, namely aplnra and $a p l n r b$, under disrupted flow conditions. At baseline, aplnrb expression was localized to the intersegmental vessels (ISVs) of $48-50 \mathrm{~h}$ post fertilization (hpf) embryos, as determined by in situ hybridization (Fig. 2f,g). Following treatment of the embryos with nifedipine, which is known to impede blood flow ${ }^{28}$, or by injection of the silent heart (sih) morpholino ${ }^{29}$, there was a significant decrease in aplnrb expression in the ISVs; demonstrating in vivo that blood flow is a critical regulator of aplnrb expression (Fig. 2f,g). The ISV vascular structure was unaffected, as confirmed by ve-cadherin staining. Aplnra expression in the ISVs, which was at baseline markedly lower than aplnrb, was either slightly increased or unchanged by nifedipine treatment or sih morpholino injection, respectively (Supplementary Fig. 10a,b).

APLN/APLNR regulation of CXCR4 is miR-139-5p dependent. Previous studies have demonstrated that shear stress can inhibit endothelial CXCR4 expression ${ }^{30}$. We found that the shearinduced downregulation of CXCR4 was abrogated by concurrent knockdown of APLNR (Fig. 3a,b), suggesting that APLNR signalling is a critical mediator of flow-induced CXCR4 inhibition. Given the inverse relation between endothelial expression of APLNR and CXCR4, we sought to determine the mechanism by which APLNR regulates CXCR4 expression. Multiple mechanisms are known to affect CXCR4 expression, including emerging data suggesting an important role for miRNA-mediated regulation in different contexts such as breast $^{31}$, prostate ${ }^{32}$, colon ${ }^{33}$ and thyroid ${ }^{34}$ cancer. The role of miRNAs as potential regulators of CXCR4 expression in ECs has not been thus far investigated. We first determined whether miRNAs in general may be playing a role in regulation of CXCR4 in ECs. We subjected HUVECs to Argonaute 2 (AGO2) knockdown, a key protein of the RNA-induced silencing complex, and found a significant increase in CXCR4 expression, suggesting that basal expression of CXCR4 may be miRNA regulated (Fig. 3c). We additionally found that shear-induced downregulation of CXCR4 was also abrogated by concurrent knockdown of $A G O 2$ (Fig. 3d), demonstrating that a miRNAbased mechanism may be critical in the regulation of CXCR4 expression in the maturing vasculature.

We next set out to identify the miRNA that may be instrumental in APLN/APLNR-mediated CXCR4 regulation. We previously conducted a miRNA expression profiling array using ECs subjected to APLN and/or APLNR knockdown, with identification of multiple miRNAs that are differentially regulated in this context ${ }^{35}$. Of the miRNAs that were identified in this setting, one in particular, namely miR-139-5p, is predicted to bind the $3^{\prime}$ untranslated region (UTR) of CXCR4 at two independent loci (Supplementary Fig. 11a,b), and was significantly downregulated by disruption of endothelial APLN/APLNR signalling ${ }^{35}$. To further investigate these relationships in vivo, we evaluated the expression levels of miR-139-5p in isolated retinal ECs from the eyes of P5 $\mathrm{Apln}^{-1-}$ and $A p l n r^{-1-}$ mice. We found significantly decreased miR-139-5p levels in fluorescence-activated cell-sorted retinal ECs from Apln ${ }^{-1-}$ and Aplnr ${ }^{-1-}$ mice compared with wildtype littermates (Fig. 3e). To further elucidate the association between miR-139-5p and APLN/APLNR signalling, we evaluated the recently described Aplnr-CreERT; $m T m G$ mice ${ }^{36}$, which express green fluorescent protein (GFP) driven by the Aplnr promoter. Expression of miR-139-5p in vivo was significantly enriched in GFP + retinal cells at P5, further demonstrating the association between APLN/APLNR signalling and miR-139-5p expression (Supplementary Fig. 12). In addition, we found that APLNR knockdown decreases the transcripts of both PDE2A, the host gene for miR-139-5p, as well as pri-miR-139, the primary transcript of miR-139-5p (Supplementary Fig. 13), suggesting a direct transcriptional relationship between APLNR and miR-139-5p. We next tested the efficacy of miR-139-5p in regulating endothelial CXCR4 expression. Overexpression of miR-139-5p in HUVECs resulted in significant decrease in the protein levels of CXCR4 (Fig. 3f). Conversely, we found that inhibition of miR-139-5p via an anti-miR inhibitor resulted in increased CXCR4 protein levels (Fig. 3f). Moreover, a luciferase reporter activity containing the wild-type CXCR4 $3^{\prime}$ UTR was significantly decreased by mir-139-5p overexpression in HUVECs, whereas the same construct with mutagenized miR-139-5p binding sites was unaffected (Supplementary Fig. 14).

Endothelial CXCR4 is closely regulated by miR-139-5p. To further elucidate the relevance of our findings to the developing vascular context, we examined whether there is a relationship between shear stress and miR-139-5p. We found that miR-139-5p was significantly induced by exposure of ECs to shear stress (Fig. 3g). Moreover, upregulation of miR-139-5p in response to shear exposure was abrogated by concurrent knockdown of APLNR, demonstrating the key role of APLNR in mediating shear response in ECs (Fig. 3g). In addition, shear-stress-mediated decrease in CXCR4 expression was abrogated by concurrent inhibition of miR-139-5p (Fig. 3h).

We carried out additional functional studies to investigate the role of miR-139-5p in ECs. Sprouting of ECs in response to SDF- $1 \alpha$ was significantly reduced in ECs overexpressing miR-139-5p, whereas sprouting in response to VEGF 165 was unaffected (Fig. 3i). Similarly, HUVECs transfected with an anti-miR against miR-139-5p sprouted more efficiently in response to SDF- $1 \alpha$ compared with control transfected cells, while VEGF-induced sprouting was not affected by anti-miR transfection (Supplementary Fig. 15a). Moreover, cell migration in response to SDF-1 $\alpha$ was also significantly impeded in HUVECs overexpressing miR-139-5p (Fig. 3j), whereas HUVECs transfected with anti-miR-139-5p had enhanced migration to SDF- $1 \alpha$ stimulation (Supplementary Fig. 15b). The migratory response to VEGF 165 was not affected by anti-miR-139-5p (Supplementary Fig. 15b).

Next, we further validated our findings in vivo, using the recently described $7 \mathrm{C} 1$ nanoparticles that are capable of selectively targeting oligonucleotide delivery to the endothelium ${ }^{37}$ Intravenous injection of anti-miR-139-5p loaded $7 \mathrm{Cl}$ nanoparticles led to partial phenocopying of the $\mathrm{Apln}^{-1-}$ and $A p l n r^{-/-}$phenotype, with significantly reduced vascularized area and radial expansion and trend towards reduced number of branchpoints, compared with mice injected with 7C1 loaded with non-targeting control (cel-miR-67-3p; Fig. 4a,b).

Restoring miR-139-5p-CXCR4 axis rescues Apln $^{-/-}$phenotype. To further validate the role of miR-139-5p as a key downstream element of APLN/APLNR signalling in vascular maturation, we sought to identify a pharmacological strategy to restore its endothelial expression. We screened multiple Food and Drug Administration (FDA) approved compounds to determine whether any of them can induce miR-139-5p expression. Of the six compounds examined, we found that atorvastatin, belonging to the class of 3-hydroxy-3-methyl-glutaryl-CoA reductase inhibitors ${ }^{38}$, induced the highest increase in miR-139-5p expression in HUVECs (Fig. 4c). We further investigated 
a

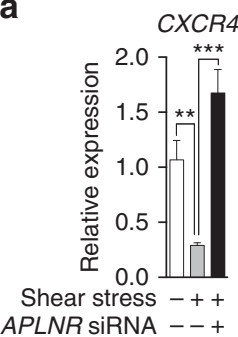

b

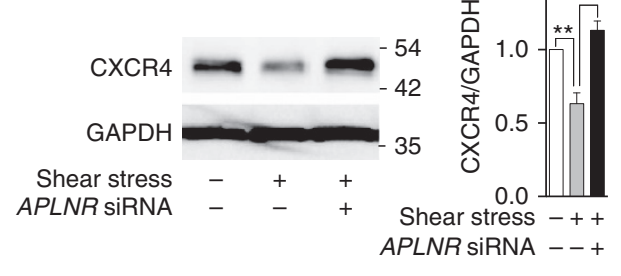

C

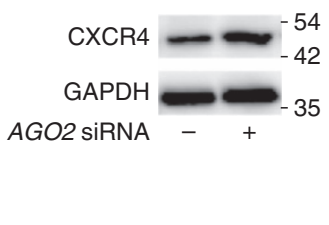

d

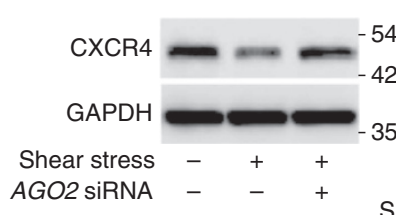

e

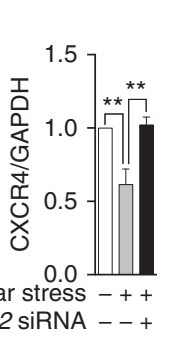

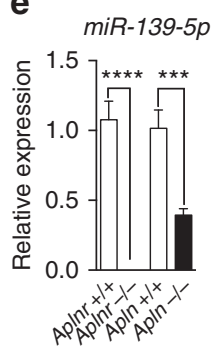

f

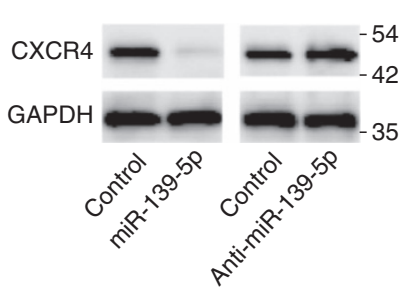

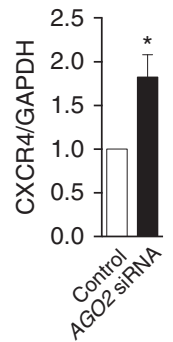

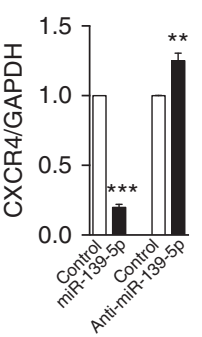

g

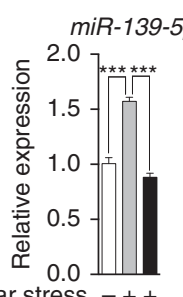

Shear stress -++ APLNR SIRNA - - +

h
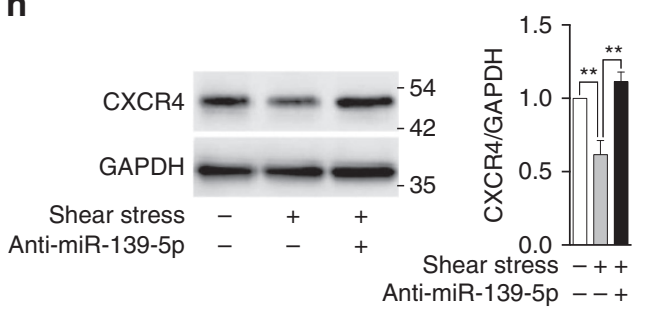

j

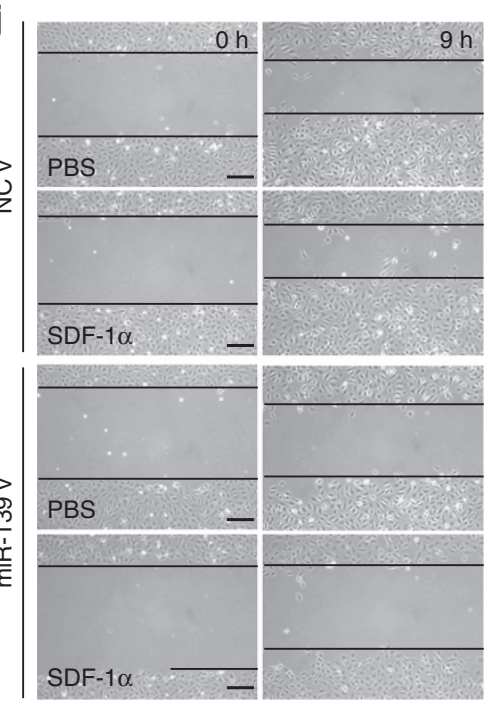

h

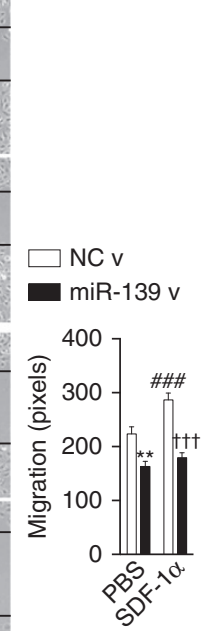

Figure 3 | APLNR regulates CXCR4 expression through miR-139-5p. (a,b) CXCR4 expression in response to shear stress exposure in HUVECs, with or without concurrent APLNR knockdown. mRNA (a) and protein levels (b) are shown. (c) CXCR4 expression in response to AGO2 knockdown. (d) CXCR4 expression in response to shear stress exposure in HUVECs, with or without concurrent AGO2 knockdown. (e) Determination of miR-139-5p levels in FACS sorted ECs from P5 retinas of $A p / n r^{-/-}$, Apln ${ }^{-/-}$mice and respective littermates. $n=3$ retinas per genotype. (f) CXCR4 expression in HUVECs overexpressing miR-139-5p mimic or non-targeting control anti-miR. (g) Expression levels of miR-139-5p in response to shear stress, with or without concurrent APLNR knockdown. (h) CXCR4 expression in HUVECs in response to shear stress exposure, with or without concurrent miR-139-5p inhibition via anti-miR. (i) Sprouting assay using HUVEC covered beads transduced with miR-139-5p or control lentivirus in response to SDF-1 $\alpha$ or VEGF 165 . Scale bar, $175 \mu \mathrm{m}$. (j) Migration assay of HUVECs transduced with miR-139-5p or control lentivirus in response to SDF- $1 \alpha$. Scale bar, $200 \mu \mathrm{m}$. ${ }^{\star} P<0.05$,

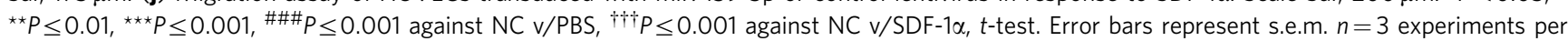
condition. 
whether atorvastatin can regulate CXCR4 expression; we found that treatment of HUVECs with atorvastatin led to marked inhibition of CXCR4 expression (Fig. 4d).

Our previous studies demonstrated that statins can augment APLN/APLNR signalling, in an APLNR-dependent manner ${ }^{39}$. Given the present data, we sought to investigate whether atorvastatin can potentially rescue the retinal vascular phenotype of mice with Apln or Aplnr deficiency. We found that atorvastatin treatment of $A$ pln ${ }^{-1-}$ mice led to robust improvement in retinal vascularized area, radial expansion and increased the number of branchpoints (Fig. 4e,f). The phenotypic rescue of either the Aplnr ${ }^{-1-}$ or the Aplnrfl/fl;Cdh5-CreERT2 mice were more modest (Supplementary Fig. 16a,b), likely in part due to the more severe phenotype of these mice, and supporting our previous data that endothelial signalling cascades induced by statin are at least in part APLNR dependent ${ }^{39}$.

\section{Discussion}

Vascular development and maturation is a complex process involving crosstalk among multiple independent, yet closely integrated signalling cascades. VEGF and Notch signalling pathways have been extensively characterized in this context as key factors that provide an intricate orchestration of endothelial identities and promote vascular network formation ${ }^{2}$. A distinct set of signalling pathways whose characterization remains at a relative infancy in this context is that mediated by GPCRs. The a
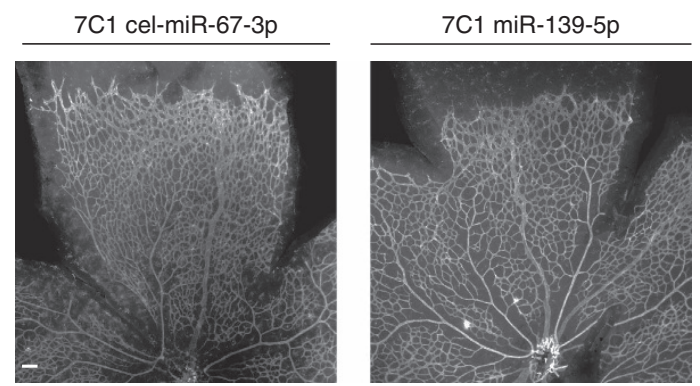

b

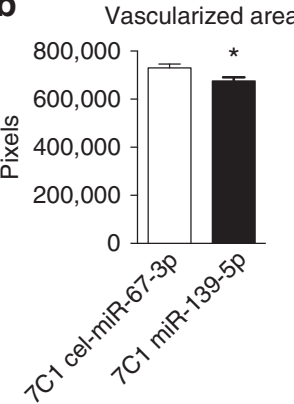

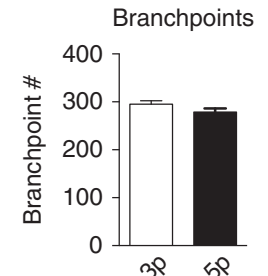

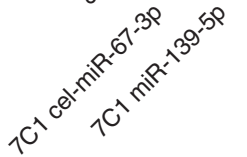

Radial expansion

C

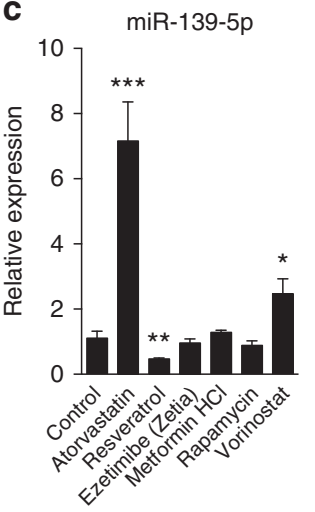

d

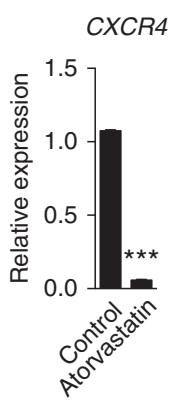

e
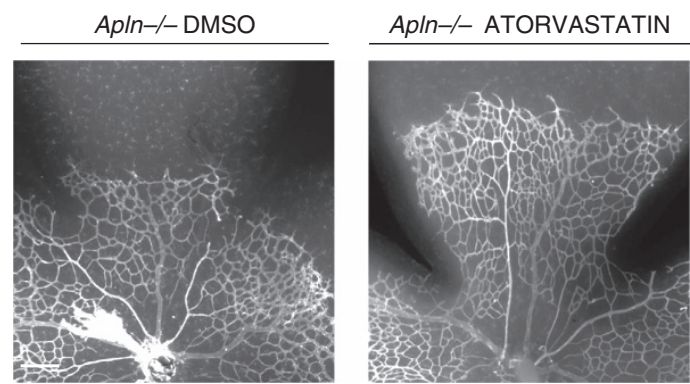

f
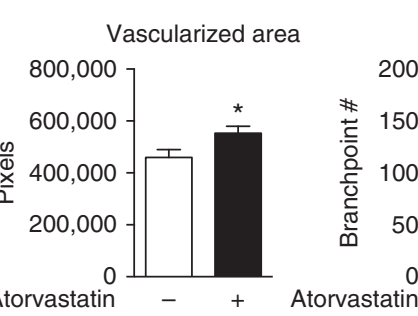

Branchpoints

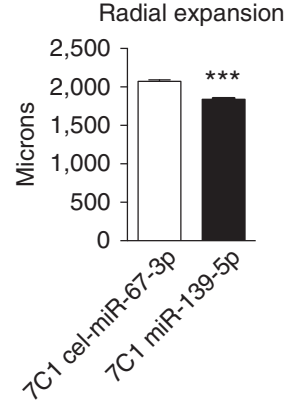

g
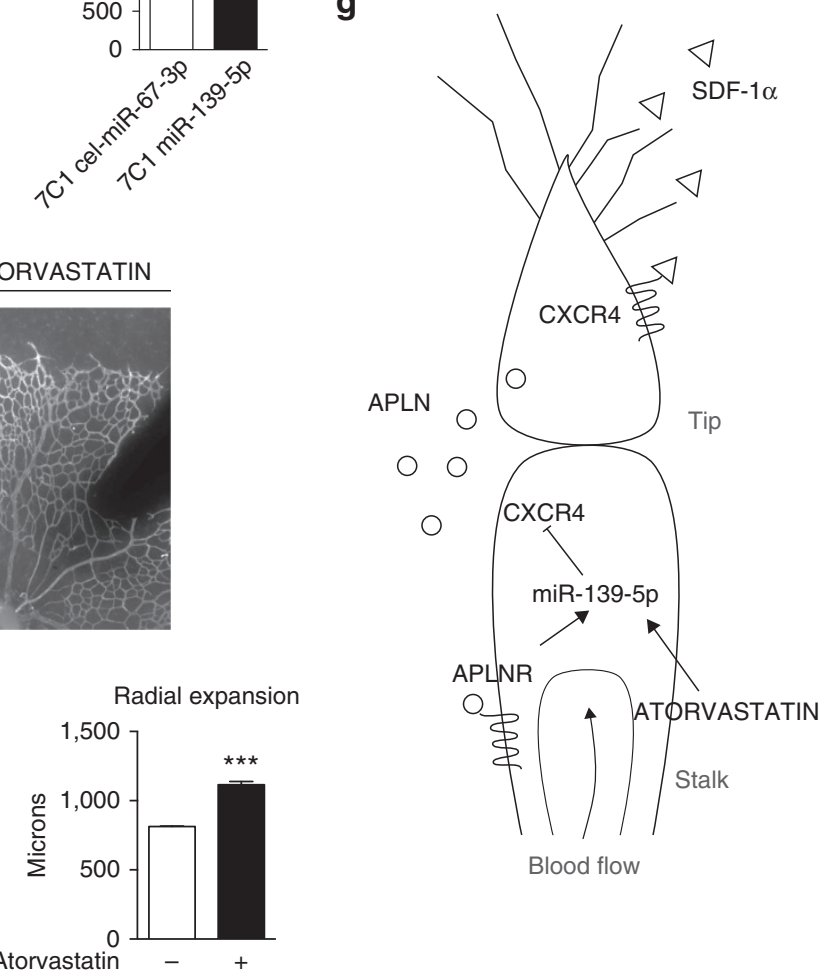

Figure 4 | Atorvastatin augments apelin/APLNR signalling and improves the $\boldsymbol{A p l n}^{-/-}$retinal phenotype. (a,b) In vivo inhibition of miR-139-5p postnatally with 7C1 nanoparticles loaded with anti-miR to miR-139-5p results in reduced vascularized area and radial expansion of retinal vasculature at P5. $n=6$ retinas per genotype. (c) Drug screen using clinically approved compounds that affect miR-139-5p expression levels in HUVECs. $n=3$ experiments. (d) CXCR4 mRNA expression in HUVECs in response to atorvastatin stimulation. $n=3$ experiments. (e,f) P5 retinal phenotype of $A p / n^{-/-}$ pups in response to treatment with atorvastatin. Vascularized area, vascular branchpoints and radial expansion are shown. Scale bar, $200 \mu \mathrm{m}$. $n \geq 4$ retinas per genotype. (g) Schematic representation of the proposed signalling cascade between APLNR, CXCR4 and the respective ligands in the developing vasculature. ${ }^{\star} P<0.05,{ }^{\star \star} P \leq 0.01,{ }^{\star \star \star} P \leq 0.001, t$-test. Error bars represent s.e.m. 
importance of GPCR signalling in vascular development is highlighted by the embryonic lethal phenotypes of several GPCRs and their respective ligand knockout mice, described to display a spectrum of cardiovascular defects $9,11,14,40-43$. However, their downstream targets and interacting partners remain incompletely defined. Adding to the complexity are emerging studies that demonstrate GPCR activation by mechanical forces such as cellular stretch and shear stress in a ligand-independent manner, which may in part explain the phenotypic differences between ligand and receptor knockout mice ${ }^{44-46}$.

CXCR4 is a GPCR with widespread functional roles in a number of contexts, including angiogenesis, hematopoiesis, neurogenesis and immune function ${ }^{47}$. In the context of vascular development, it has been established as a tip cell-specific gene in the developing mouse and zebrafish vasculature ${ }^{10,48}$. Interestingly, it is one of a few tip cell enriched endothelial genes that is also known to be markedly downregulated by shear stress in vitro ${ }^{30}$, and by blood flow in the zebrafish in vivo ${ }^{48,49}$. These findings suggest that CXCR4 needs to be strictly regulated in the developing vasculature, to restrict the cellular response to its ligands such as SDF- $1 \alpha$, and avoid the potential detriment of inappropriate EC behaviour resulting from aberrantly expressed CXCR4. Although this possibility has not been specifically examined in the vascular context, whole embryo overexpression of Cxcr4a in zebrafish results in significant craniofacial and cartilage defects, highlighting the requirement for stringent CXCR4 regulation ${ }^{50}$. In addition, although multiple studies have suggested pro-angiogenic effects of augmenting CXCR4 signalling $49,51,52$, its restricted expression pattern in the developmental context ${ }^{10,48}$ suggests the importance of limiting expression to endothelial subsets for normal angiogenesis. Mechanisms that regulate its expression, however, remain inadequately defined. The current body of work provides key insights into a novel mechanism that integrates flow, a secondary GPCR signalling mediated by APLN/APLNR, and miR-139-5p, to restrict endothelial CXCR4 expression in the context of vascular development and maturation (Fig. $4 \mathrm{~g}$ for schematic).

The abnormal vascular patterning of Apln and Aplnr null mice likely involves a multitude of downstream signalling targets, some of which have been described previously in other endothelial contexts $^{11}$. Our findings that CXCR4 expression is decreased by APLNR-mediated signalling, and that inhibition of CXCR4 activity with AMD3100 can achieve a partial rescue of the Aplnr ${ }^{-/-}$and Aplnrfl/fl;Cdh5-CreERT2 phenotype highlights the importance of this mechanism, but also implicate other downstream targets that are also likely involved in regulating processes such as cellular proliferation and migration, lumen formation and maintenance, and regulation of vascular permeability ${ }^{53}$. Ongoing studies will further delineate the role of this and other related GPCR signalling cascades in vascular maturation.

Onset of blood flow, and subjecting ECs to shear stress leads to a remarkable alteration in the endothelial behaviour and function. Multiple in vivo models have demonstrated the role of flow in vascular development in murine ${ }^{54,55}$, avian ${ }^{56}$ and fish models ${ }^{57}$. A number of shear responsive endothelial elements have also been identified, including kinases (ERK5 (ref. 58), ERK1/2 (ref. 59) and AKT (ref. 60)), transcription factors (KLF2 (ref. 61), and KLF4 (ref. 62)) and other epigenetic modifiers (miR-126 (ref. 57)). Our current findings highlight the induction of APLNR expression in ECs subjected to shear stress, an effect that was dependent at least in part on ERK5, KLF2 and KLF4, which are genes with key flow responsive roles in the endothelium.

The specific characterization of the flow pattern in the developing vascular bed remains incompletely defined, although recent studies have used computational analyses to estimate the shear profiles ${ }^{23}$. We found induction of APLNR in ECs in response to multiple types of shear stress, and furthermore showed that aplnrb, one of the two paralogous apln receptors in the zebrafish, was markedly decreased with cessation of blood flow.

miRNAs are emerging as vital rheostats in multiple biological processes, and their key roles in the context of vascular development are only beginning to be appreciated. The intricacies of miRNA-mediated signalling integration in vascular development remain at infancy, although recent studies have demonstrated their key roles in angiogenesis ${ }^{57}$. Although miR-139-5p has been described in a limited number of studies as a potential tumour suppressor miRNA ${ }^{63,64}$, little is known about the function or regulation of $\mathrm{miR}-139-5 \mathrm{p}$ in the endothelium. Our current findings demonstrate two key aspects of this highly regulated endothelial miRNA that is likely a critical mediator of vascular maturation. First, we identified miR-139-5p as a novel shear responsive endothelial miRNA, that is APLN/APLNR dependent. Second, we found that miR-139-5p is a key intermediary that links APLN/APLNR signalling with repression of endothelial CXCR4 expression, which appears to be essential for proper vascular maturation. Lastly, we show that in vivo inhibition of miR-139-5p postnatally results in partial phenocopying of the Apln ${ }^{-1-}$ and Aplnr ${ }^{-1-}$ phenotypes. We expect that our ongoing studies into the role of miR-139-5p in the endothelium, both in the developmental and the vascular disease context, will shed greater insights into its functions.

A key aspect of our current findings is the effect of statins in induction of miR-139-5p expression, inhibition of CXCR4 expression and pharmacological rescue of the retinal phenotype of the $A p \ln ^{-1-}$ mice. These findings provide support for our previous findings describing the augmentation of APLNRmediated signalling by statins ${ }^{39}$, the mechanism of which will require further investigation. From the perspective of statin pleiotropy, these findings also invoke new potential endothelial targets of statins, which may be playing a key role in mediating the vascular protective effects of these agents in atherosclerosis.

In summary, the crosstalk between APLNR and CXCR4mediated pathways provide a novel mechanism that may be a crucial determinant of vascular maturation. Further studies to expand on these findings, as well as identification of potential relation between these elements and the role of VEGF-Notch signalling cascade, will advance our understanding of the signalling crosstalk involved in vascular maturation.

\section{Methods}

Animals. All animal experiments were conducted in compliance with the relevant laws and institutional guidelines and were approved by Yale University Institutional Animal Care and Use Committee and local animal ethics committees of the Landesamt für Natur, Umwelt und Verbraucherschutz Nordrhein-Westfalen. Aplnr ${ }^{-1-}$, Aplnrfl/fl, Apln ${ }^{-/-}$, Cdh5-CreERT2 and Aplnr-CreER; Rosa ${ }^{\text {mTmG }}$ mice have been previously described ${ }^{19,36,65}$. All animals were maintained on a C57Bl/6 background, except for Aplnr-CreER; Rosa ${ }^{m T m G}$ which were on a mixed background. Given the young age of the mice at the time of our studies, their gender was not determined. To inhibit CXCR4, $5 \mathrm{mg} \mathrm{kg}^{-1}$ of AMD3100

(Sigma-Aldrich) dissolved in PBS were injected intraperitoneally in mice at P0 and P1. Injection with PBS was used as negative control. Atorvastatin (Cayman Chemical) was dissolved in dimethyl sulfoxide (DMSO) at $15 \mathrm{mg} \mathrm{ml}^{-1}$ and further diluted in PBS. Mice were injected intraperitoneally at P1, P2 and P3 with $40 \mathrm{mg} \mathrm{kg}^{-1}$. Injection with PBS/DMSO was used as negative control. To induce recombination of the Aplnr allele with the Cdh5-CreERT2 driver or recombination of Rosa ${ }^{m T m G}$ with the Aplnr-CreER driver, mice received intragastric injection of $100 \mu \mathrm{g}$ of Tamoxifen (Sigma-Aldrich) dissolved in corn oil (Sigma-Aldrich) at P1 and P2. FITC Dextran (MW $=2 \times 10^{6} \mathrm{Da}$; Sigma-Aldrich) was dissolved in water and injected in the left ventricle of anaesthetized P5 pups and allowed to perfuse for $1 \mathrm{~min} .7 \mathrm{C} 1$ nanoparticles ${ }^{37}$ containing custom made miRIDIAN Hairpin inhibitors for miR-139-5p or cel-miR-67-3p (Dharmacon) were injected daily intravenously at $1 \mathrm{mg} \mathrm{kg}^{-1}$ at $\mathrm{P} 0-\mathrm{P} 2$, via the superficial temporal facial vein ${ }^{66}$. All mice for retinal vasculature studies were killed at $\mathrm{P} 5$.

For zebrafish blood flow blocking experiments, a stock solution of $10 \mathrm{mM}$ nifedipine (Sigma-Aldrich) in DMSO was diluted 1:4,000 (working concentration $2.5 \mu \mathrm{M}$ ) in $\mathrm{E} 3$ medium (diluted from a $60 \times$ stock containing $34.8 \mathrm{~g} \mathrm{NaCl}, 1.6 \mathrm{~g}$ 
$\mathrm{KCl}$, $5.8 \mathrm{~g} \mathrm{CaCl}_{2} \times 2 \mathrm{H}_{2} \mathrm{O}, 9.78 \mathrm{~g} \mathrm{MgCl} \times 6 \mathrm{H}_{2} \mathrm{O}$ dissolved in $21 \mathrm{H}_{2} \mathrm{O}$ and $\mathrm{pH}$ adjusted to 7.2 with $\mathrm{NaOH}$ ) containing $2 \times$ tricaine and $0.003 \%$ phenylthiourea to prevent pigmentation. Tricaine $(2 \times)$ was prepared by adding $8.4 \mathrm{ml}$ of a stock solution ( $400 \mathrm{mg}$ tricaine powder, $97.9 \mathrm{ml} \mathrm{H}_{2} \mathrm{O}, 2.1 \mathrm{ml}$ Tris (pH9), $\mathrm{pH}$ adjusted to $7.0)$ to $100 \mathrm{ml}$ of $\mathrm{E} 3$ medium. Embryos were incubated for $1 \mathrm{~h}$ and then fixed in $4 \%$ paraformaldehyde (PFA) for in situ hybridization experiments. For washout experiments, embryos were washed once with E3 medium, and then transferred to E3 medium without nifedipine.

Morpholinos targeting cardiac troponin T2 (silent heart (sih), 2 ng per embryo) were designed to bind the translation start codon and flanking $5^{\prime}$ sequence (5'-CATGTTTGCTCTGATCTGACACGCA- $\left.3^{\prime}\right)$ and injected into embryos at the $1-4$ cell stage.

\section{Immunostaining and in situ hybridization. Isolectin B4 staining (Life} Technologies, working concentration $20 \mu \mathrm{g} \mathrm{ml}^{-1}$ ) was performed at room temperature for $1 \mathrm{~h}$ or at $4{ }^{\circ} \mathrm{C}$ overnight. Erg123 (ab92513, Abcam, 1/100) antibody staining was performed at $4^{\circ} \mathrm{C}$ overnight. Alexa Fluor (Life Technologies, working concentration $10 \mu \mathrm{g} \mathrm{ml}^{-1}$ ) secondary antibody was used. Retinal analysis of the different mice was performed on at least four mice per genotype. Retinas were analysed with a Nikon Eclipse 80i microscope, equipped with a Retiga 2000R Fast1394 camera. NIS Elements software was used for image acquisition and Adobe Photoshop CS5.1 and ImageJ and Matlab were used for image processing. For quantification of vascularized area, vascular branchpoints and radial expansion of the retinas at least five images per retina were acquired at $\times 20$ magnification at the tip-stalk cell border and between arteries and veins.

For in situ hybridization eyes from P5 pups were fixed in 4\% PFA for $30 \mathrm{~min}$ at $4{ }^{\circ} \mathrm{C}$, before the retinas were collected and fixed again in $4 \% \mathrm{PFA}$ at $4{ }^{\circ} \mathrm{C}$ overnight. Retinas were dehydrated in methanol series and stored at $-20^{\circ} \mathrm{C}$ until required. Before hybridization, retinas were rehydrated to $\mathrm{PBS} / 0.1 \%$ Tween and then digested for $11 \mathrm{~min}$ in $80 \mu \mathrm{g} \mathrm{ml}^{-1}$ Proteinase K (Sigma-Aldrich) followed by fixation in $4 \% \mathrm{PFA} / 0.2 \%$ glutaraldehyde in PBS/0.1\% Tween. Retinas were washed in $\mathrm{PBS} / 0.1 \%$ Tween, pre-incubated in hybridization buffer at $70^{\circ} \mathrm{C}$ for $3 \mathrm{~h}$ and then incubated with RNA probes in hybridization buffer at $70^{\circ} \mathrm{C}$ overnight. Probes were labelled with digoxigenin-uridine triphosphate (Roche) using full-length complementary DNA (cDNA) clones and T7, T3 or Sp6 polymerases. The Apln plasmid was a kind gift from A. Eichmann ${ }^{12}$, Aplnr and Cxcr4 plasmids were purchased from Dharmacon (MMM1013-202766118, clone ID: 4457726, MMM1013-202762695, clone ID: 3592479). After extensive washing the hybridized probes were detected with alkaline phosphatase-conjugated anti-digoxigeninalkaline phosphatase antibody (11093274910, Roche, working concentration $0.375 \mathrm{U} \mathrm{ml}^{-1}$ ) at $4{ }^{\circ} \mathrm{C}$ overnight and signal was visualized with $\mathrm{BM}$ purple alkaline phosphatase substrate (1144207001, Roche). Retinas were mounted using Vectashield (Vector Labs) and imaged.

For zebrafish in situ hybridization, aplnra and aplnrb probes were amplified from cDNA of 19 somite stage-old embryos using the following primers: aplnraforward 5'-GAAAGGCCCAAGTCACAGAG-3' and aplnra-reverse 5'-GAGTTC ACTATCTGATGTCAAACCA- $3^{\prime}$, apln rb-forward $5^{\prime}$-GAAAGGCCCAAGTCAC AGAG- $3^{\prime}$ and $a p l n r b$-reverse $5^{\prime}$-GAGTTCACTATCTGATGTCAAACCA- $3^{\prime}$. The T7 promotor was added to the $5^{\prime}$ end of the reverse primer in a second round of amplification (T7-aplnra-reverse 5'-GTAATACGACTCACTATAGGGAGTTCA CTATCTGATGTCAAACCA-3', T7-aplnrb-reverse 5'-GTAATACGACTCACT ATAGGGAGTTCACTATCTGATGTCAAACCA- $\left.3^{\prime}\right)^{67}$. The ve-cadherin probe was synthesized using a plasmid containing a 1,395-bp $c d h 5$ DNA segment. mRNA was synthesized in vitro using digoxigenin-uridine triphosphate (Roche) and T7 or T3 Polymerase (Roche).

Cells and reagents. HUVECs (Yale VBT Core) were cultured at $37^{\circ} \mathrm{C}$ in a $5 \%$ $\mathrm{CO}_{2}$ incubator in EGM-2 medium (Lonza). For experimental treatments, HUVECs (passages 3-7) were grown to 70-90\% confluence. Laminar or pulsatile shear stress was applied to confluent cell cultures at 12 or $12 \pm 5$ dynes $\mathrm{cm}^{-2}$, respectively, using a parallel plate chamber. A surface area of $14 \mathrm{~cm}^{2}$ on the HUVEC-seeded slide was exposed to fluid shear stress generated by perfusing culture medium over the cells. The $\mathrm{pH}$ of the system was kept constant by gassing with $5 \% \mathrm{CO}_{2} / 95 \%$ air and the temperature was maintained at $37^{\circ} \mathrm{C}$. Orbital shear stress, calculated at 10.7 dynes $\mathrm{cm}^{-2}$, was applied to confluent cell cultures seeded in 6-well plates, using an orbital shaker positioned inside an incubator. Orbital shear stress was used for all shear experiments (other than alternate conditions for Supplementary Fig. 7). Transient transfections of plasmids were performed with Fugene HD (Promega) according to the manufacturer's instructions. For gene silencing, short interfering RNAs (siRNAs; Stealth siRNA, Invitrogen) were transfected with RNAiMAX (Invitrogen) according to the manufacturer's instructions. Non-targeting control and anti-miR-139-5p oligonucleotides (Dharmacon) were transfected with RNAiMAX (Invitrogen) according to the manufacturer's instructions. Control and miR-139 lentivirus constructs (System Biosciences) were used to generate lentivirus particles with the Lenti-X HTX Packaging System (Clontech) with Lenti-X Concentrator. For in vivo inhibition of miR-139-5p, custom made miRIDIAN Hairpin inhibitors for miR-139-5p or cel-miR-67-3p were used (Dharmacon) for packaging in the $7 \mathrm{C} 1$ nanoparticles ${ }^{37}$. To generate 7C1 nanoparticles, polyethyleneimine with a number molecular weight of 600 (PEI600, Sigma-Aldrich) was combined with 200 proof anhydrous ethanol
(Koptec) and an epoxide-terminated C15 lipid at a lipid:PEI molar ratio equal to 14:1. The mixture was heated at $90^{\circ} \mathrm{C}$ for $48 \mathrm{~h}$ before purification was performed with a silica column ${ }^{37}$. To formulate nanoparticles, purified $7 \mathrm{C} 1$ was combined with 200 proof ethanol and (1,2-dimyristoyl-sn-glycero-3-phosphoethanolamine$N$-[methoxy(polyethylene glycol)-2000] (Avanti Polar Lipids) at a 7C1:lipidpolyethylene glycol (PEG) molar ratio equal to 4:1 in a glass syringe. siRNA was dissolved in $\mathrm{pH} 310 \mathrm{mM}$ citrate solution (Teknova) in a separate syringe. The two syringes were connected to a syringe pump and the fluid was pushed through a microfluidic device. The resulting nanoparticles were dialyzed in $1 \times$ PBS and filtered through a $0.22-\mu \mathrm{m}$ sieve before their size was characterized.

HUVECs were treated for $24 \mathrm{~h}$ with atorvastatin (working concentration $10 \mu \mathrm{M}$ in DMSO), vorinostat (working concentration $10 \mu \mathrm{M}$ in PBS), resveratrol (working concentration $10 \mu \mathrm{M}$ in PBS), ezetimibe (working concentration $10 \mu \mathrm{M}$ in PBS), metformin (working concentration $100 \mu \mathrm{M}$ in $\mathrm{H}_{2} \mathrm{O}$ ) or rapamycin (working concentration 20nM in EtOH; Cayman Chemical) with the corresponding controls.

Cell migration assay. HUVECs were incubated in 6-well plates in EGM-2 medium overnight. After serum starvation for $6 \mathrm{~h}$ with EBM-2 medium, the monolayer of HUVECs was scratched with a universal blue pipette tip and the widths of the scratches in four fields per well were captured using a Zeiss microscope with a $\times 10$ objective. Cells were incubated for $9 \mathrm{~h}$ in starvation medium containing PBS and $50 \mathrm{ng} \mathrm{ml}^{-1}$ SDF-1 $\alpha$ (SRP3252, Sigma-Aldrich). The same fields were captured again after migration. Differences in the widths of scratches before and after migration were calculated. The calculated width is an average of the widths of three measurements taken from the same field. The means and s.e.m. of triplicate wells were calculated. All experiments were conducted at least three times.

Fibrin gel bead assay. HUVECs were mixed with Cytodex 3 microcarriers (Amersham Pharmacia Biotech) at a concentration of 400 HUVECs per bead in $1 \mathrm{ml} \mathrm{EGM}-2$ medium. Beads with cells were shaken gently every $20 \mathrm{~min}$ for $4 \mathrm{~h}$ at $37^{\circ} \mathrm{C}$ in $5 \% \mathrm{CO}_{2}$. After incubation, the beads with cells were transferred to a $25 \mathrm{~cm}^{2}$ tissue culture flask and left for $12-16 \mathrm{~h}$ in $5 \mathrm{ml} \mathrm{EGM-2}$. The following day, these were washed three times with $1 \mathrm{ml} \mathrm{EGM-2}$ and resuspended at a concentration of 200 cell-coated beads per $\mathrm{ml}$ in $2.5 \mathrm{mg} \mathrm{ml}^{-1}$ fibrinogen (Sigma-Aldrich) containing $0.15 \mathrm{U} \mathrm{ml}^{-1}$ aprotinin (Sigma-Aldrich). A total of $500 \mu \mathrm{l}$ fibrinogen/bead solution was added to $0.625 \mathrm{U}$ of thrombin (Sigma-Aldrich) in one well of a 24-well tissue culture plate. The fibrinogen/bead solution was allowed to clot for $5 \mathrm{~min}$ at room temperature and then at $37^{\circ} \mathrm{C}$ in $5 \% \mathrm{CO}_{2}$ for $15 \mathrm{~min}$. EGM-2 containing $50 \mathrm{ng} \mathrm{ml}^{-1}$ SDF-1 $\alpha$ (SRP3252, Sigma-Aldrich), or $20 \mathrm{ng} \mathrm{ml}^{-1}$ VEGF 165 (293-VE, R\&D Systems) was used. Fibroblasts were layered on top of the clot at a concentration of 20,000 cells per well. The medium was changed every other day, and SDF- $1 \alpha$ and VEGF 165 were added daily.

3' UTR luciferase reporter assay. Human CXCR4 $3^{\prime}$ UTR reporter construct was purchased from SwitchGear Genomics. For mutant analysis, the sequence $5^{\prime}$-CTG TAG-3' in the two predicted miR-139-5p binding sites was mutated to $5^{\prime}$-GGA TCC- $3^{\prime}$. HUVECs were transfected with the luciferase reporter constructs containing the CXCR4 $3^{\prime}$ UTR variants, Renilla luciferase and $30 \mathrm{nM}$ of either miR-139-5p mimic or negative control miRNA. At $24 \mathrm{~h}$ after transfection, the cells were lysed, and luciferase activity was measured using the Dual-Luciferase Reporter Assay kit (Promega) according to the manufacturer's instructions.

Flow cytometry. Up to four retinas of wild type or Aplnr ${ }^{-/-}$P5 mouse neonates were pooled and incubated for $15 \mathrm{~min}$ at $37^{\circ} \mathrm{C}$ in EBM-2 containing Collagenase A $\left(1.5 \mathrm{mg} \mathrm{ml}^{-1}, 103578\right.$, Roche $)$ and DNAase I $\left(25 \mu \mathrm{g} \mathrm{ml}{ }^{-1}, 104159\right.$, Roche $)$ before cells were dissociated by gentle trituration. Cells were stained with FITCconjugated anti-CD31 antibody (558738, BD Biosciences, working concentration $5 \mu \mathrm{g} \mathrm{ml}^{-1}$ ) or APC-conjugated anti-CXCR4 antibody (558644, BD Biosciences, working concentration $2 \mu \mathrm{g} \mathrm{ml}^{-1}$ ). Stained cells were sorted using a FACSAria flow cytometer (BD Biosciences) or an LSRII flow cytometer (BD Biosciences). When cells were collected, both the negative and positive selections were collected in ice cold PBS and processed for RNA extraction and real-time (RT) PCR as described below. Data quantification was performed with FlowJo 7.6 software.

HUVECs transfected with GFP or APLNR-GFP (Origene) plasmids for $48 \mathrm{~h}$ were detached from the culture dish, washed and subsequently resuspended with PBS containing $5 \%$ FBS. The cells were incubated at $4{ }^{\circ} \mathrm{C}$ for $30 \mathrm{~min}$ with phycoerythrin-conjugated CXCR4 antibody (551510, BD Biosciences, working concentration $2 \mu \mathrm{g} \mathrm{ml}^{-1}$ ). Cells were washed with PBS and CXCR4 expression was analysed by flow cytometry (BD FACScan). Data quantification was performed with FlowJo 7.6 software.

RNA analysis. Total RNA was extracted using the miRNeasy RNA isolation kit (Qiagen). Purified RNA was reverse transcribed with the iScript cDNA Synthesis Kit (Bio-Rad) or the TaqMan MicroRNA Reverse Transcription Kit (Life Technologies). RT-PCR was performed with TaqMan probes for both genes and miRNAs (Life Technologies). RT-PCR for miR-139-5p detected both the human (hsa-miR-139-5p) and murine (mmu-miR-139-5p) variants. All miRNA data were normalized to the internal control small RNAs RNU19 or U6. For the 
mRNA samples, ribosomal $18 \mathrm{~S}$ was used as internal control. Individual RT-PCRs were performed on a CFX96 (Bio-Rad) according to the manufacturer's instructions. TaqMan probes were used for all genes.

Protein extraction and western blotting. HUVECs were lysed with RIPA lysis buffer (Thermo Scientific) containing Halt Protease and Phosphatase Inhibitor cocktail (Thermo Scientific). Total protein contents of soluble cell lysate were measured using a Micro BCA Protein assay kit (Thermo Scientific). Western blotting was performed using standard methods. Briefly, total protein from each sample was resolved by sodium dodecyl sulfate-polyacrylamide gel electrophoresis, transferred to a polyvinylidene difluoride membrane (Bio-Rad) and probed with antibodies specific to CXCR4 (ab2074, Abcam, 1:2,000) and GAPDH (2118, Cell Signaling, 1:3,000). Subsequently, the membranes were incubated with HRP-conjugated secondary antibodies (7074, Cell Signaling, 1:3,000) and developed using an enhanced chemiluminescence detection method (Thermo Scientific). Uncropped scans of key western blots are provided as a Supplementary Fig. 17.

Statistical analyses. Unless otherwise noted, all cell treatments, cell migration and sprouting assays, western blots, quantitative PCR assays and in situ hybridizations are representative of at least three independent experiments. Results are reported as mean \pm s.e.m. Unpaired Student's $t$-test was used to determine statistical significance. Post hoc analysis was performed using the Holm-Sidak method. $P<0.05$ was considered significant.

\section{References}

1. Siekmann, A. F., Affolter, M. \& Belting, H. G. The tip cell concept 10 years after: new players tune in for a common theme. Exp. Cell Res. 319, 1255-1263 (2013).

2. Blanco, R. \& Gerhardt, H. VEGF and Notch in tip and stalk cell selection. Cold Spring Harb. Perspect. Med. 3, a006569 (2013).

3. Gerhardt, H. et al. VEGF guides angiogenic sprouting utilizing endothelial tip cell filopodia. J. Cell Biol. 161, 1163-1177 (2003).

4. Hoey, T. et al. DLL4 blockade inhibits tumor growth and reduces tumor-initiating cell frequency. Cell Stem Cell 5, 168-177 (2009).

5. Hellstrom, M. et al. Dll4 signalling through Notch1 regulates formation of tip cells during angiogenesis. Nature 445, 776-780 (2007).

6. Lobov, I. B. et al. Delta-like ligand 4 (Dll4) is induced by VEGF as a negative regulator of angiogenic sprouting. Proc. Natl Acad. Sci. USA 104, 3219-3224 (2007)

7. Siekmann, A. F. \& Lawson, N. D. Notch signalling limits angiogenic cell behaviour in developing zebrafish arteries. Nature 445, 781-784 (2007).

8. Suchting, S. et al. The Notch ligand Delta-like 4 negatively regulates endothelial tip cell formation and vessel branching. Proc. Natl Acad. Sci. USA 104, 3225-3230 (2007).

9. Tachibana, K. et al. The chemokine receptor CXCR4 is essential for vascularization of the gastrointestinal tract. Nature 393, 591-594 (1998).

10. Strasser, G. A., Kaminker, J. S. \& Tessier-Lavigne, M. Microarray analysis of retinal endothelial tip cells identifies CXCR4 as a mediator of tip cell morphology and branching. Blood 115, 5102-5110 (2010).

11. Kang, Y. et al. Apelin-APJ signaling is a critical regulator of endothelial MEF2 activation in cardiovascular development. Circ. Res. 113, 22-31 (2013).

12. del Toro, R. et al. Identification and functional analysis of endothelial tip cellenriched genes. Blood 116, 4025-4033 (2010).

13. Xu, C. et al. Arteries are formed by vein-derived endothelial tip cells. Nat. Commun. 5, 5758 (2014).

14. Liu, Y. et al. Edg-1, the G protein-coupled receptor for sphingosine-1phosphate, is essential for vascular maturation. J. Clin. Invest. 106, 951-961 (2000)

15. Jung, B. et al. Flow-regulated endothelial S1P receptor-1 signaling sustains vascular development. Dev. Cell 23, 600-610 (2012).

16. Gaengel, K. et al. The sphingosine-1-phosphate receptor S1PR1 restricts sprouting angiogenesis by regulating the interplay between VE-cadherin and VEGFR2. Dev. Cell 23, 587-599 (2012).

17. Kasai, A. et al. Retardation of retinal vascular development in apelin-deficient mice. Arterioscler. Thromb. Vasc. Biol. 28, 1717-1722 (2008).

18. Sakimoto, S. et al. A role for endothelial cells in promoting the maturation of astrocytes through the apelin/APJ system in mice. Development 139, 1327-1335 (2012).

19. Wang, Y. et al. Ephrin-B2 controls VEGF-induced angiogenesis and lymphangiogenesis. Nature 465, 483-486 (2010).

20. Hatse, S., Princen, K., Bridger, G., De Clercq, E. \& Schols, D. Chemokine receptor inhibition by AMD3100 is strictly confined to CXCR4. FEBS Lett. 527, 255-262 (2002)

21. Unoki, N. et al. SDF-1/CXCR4 contributes to the activation of tip cells and microglia in retinal angiogenesis. Invest. Ophthalmol. Vis. Sci. 51, 3362-3371 (2010).
22. Saint-Geniez, M., Argence, C. B., Knibiehler, B. \& Audigier, Y. The msr/apj gene encoding the apelin receptor is an early and specific marker of the venous phenotype in the retinal vasculature. Gene Expr. Patterns 3, 467-472 (2003).

23. Bernabeu, M. O. et al. Computer simulations reveal complex distribution of haemodynamic forces in a mouse retina model of angiogenesis. J. R. Soc. Interface 11, pii: 20140543 (2014).

24. Dardik, A. et al. Differential effects of orbital and laminar shear stress on endothelial cells. J. Vasc. Surg. 41, 869-880 (2005).

25. Ohnesorge, N. et al. Erk5 activation elicits a vasoprotective endothelial phenotype via induction of Kruppel-like factor 4 (KLF4). J. Biol. Chem. 285, 26199-26210 (2010).

26. Chng, S. C., Ho, L., Tian, J. \& Reversade, B. ELABELA: a hormone essential for heart development signals via the apelin receptor. Dev. Cell 27, 672-680 (2013).

27. Pauli, A. et al. Toddler: an embryonic signal that promotes cell movement via Apelin receptors. Science 343, 1248636 (2014).

28. Langheinrich, U., Vacun, G. \& Wagner, T. Zebrafish embryos express an orthologue of HERG and are sensitive toward a range of QT-prolonging drugs inducing severe arrhythmia. Toxicol. Appl. Pharmacol. 193, 370-382 (2003).

29. Sehnert, A. J. et al. Cardiac troponin $\mathrm{T}$ is essential in sarcomere assembly and cardiac contractility. Nat. Genet. 31, 106-110 (2002).

30. Melchionna, R. et al. Laminar shear stress inhibits CXCR4 expression on endothelial cells: functional consequences for atherogenesis. FASEB J. 19, 629-631 (2005).

31. Liang, Z., Bian, X. \& Shim, H. Inhibition of breast cancer metastasis with microRNA-302a by downregulation of CXCR4 expression. Breast Cancer Res. Treat. 146, 535-542 (2014).

32. Shen, P. F. et al. MicroRNA-494-3p targets CXCR4 to suppress the proliferation, invasion, and migration of prostate cancer. Prostate 74, 756-767 (2014).

33. Li, Z. et al. Expression of miR-126 suppresses migration and invasion of colon cancer cells by targeting CXCR4. Mol. Cell. Biochem. 381, 233-242 (2013).

34. Leone, V. et al. MiR-1 is a tumor suppressor in thyroid carcinogenesis targeting CCND2, CXCR4, and SDF-1alpha. J. Clin. Endocrinol. Metab. 96, E1388-E1398 (2011).

35. Kim, J. et al. An endothelial apelin-FGF link mediated by miR-424 and miR-503 is disrupted in pulmonary arterial hypertension. Nat. Med. 19, 74-82 (2013).

36. Chen HI, S. B. et al. The sinus venosus contributes to the coronary vasculature through vegf-c stimulated angiogenesis. Development 141, 4500-4512 (2014).

37. Dahlman, J. E. et al. In vivo endothelial siRNA delivery using polymeric nanoparticles with low molecular weight. Nat. Nanotechnol. 9, 648-655 (2014)

38. Laufs, U. \& Liao, J. K. Isoprenoid metabolism and the pleiotropic effects of statins. Curr. Atheroscler. Rep. 5, 372-378 (2003).

39. McLean, D. L. et al. Apelin/APJ signaling is a critical regulator of statin effects in vascular endothelial cells--brief report. Arterioscler. Thromb. Vasc. Biol. 32, 2640-2643 (2012).

40. Loot, A. E. \& Fleming, I. A novel APJ signaling cascade that regulates cardiovascular development. Circ. Res. 113, 4-6 (2013).

41. Caron, K. M. \& Smithies, O. Extreme hydrops fetalis and cardiovascular abnormalities in mice lacking a functional Adrenomedullin gene. Proc. Natl Acad. Sci. USA 98, 615-619 (2001).

42. Dackor, R. T. et al. Hydrops fetalis, cardiovascular defects, and embryonic lethality in mice lacking the calcitonin receptor-like receptor gene. Mol. Cell. Biol. 26, 2511-2518 (2006)

43. Connolly, A. J., Ishihara, H., Kahn, M. L., Farese, Jr R. V. \& Coughlin, S. R. Role of the thrombin receptor in development and evidence for a second receptor. Nature 381, 516-519 (1996).

44. Scimia, M. C. et al. APJ acts as a dual receptor in cardiac hypertrophy. Nature 488, 394-398 (2012).

45. Rakesh, K. et al. beta-Arrestin-biased agonism of the angiotensin receptor induced by mechanical stress. Sci. Signal. 3, ra46 (2010).

46. Chachisvilis, M., Zhang, Y. L. \& Frangos, J. A. G protein-coupled receptors sense fluid shear stress in endothelial cells. Proc. Natl Acad. Sci. USA 103, 15463-15468 (2006).

47. Jacobson, O. \& Weiss, I. D. CXCR4 chemokine receptor overview: biology, pathology and applications in imaging and therapy. Theranostics 3, 1-2 (2013).

48. Bussmann, J., Wolfe, S. A. \& Siekmann, A. F. Arterial-venous network formation during brain vascularization involves hemodynamic regulation of chemokine signaling. Development 138, 1717-1726 (2011).

49. Packham, I. M. et al. Microarray profiling reveals CXCR4a is downregulated by blood flow in vivo and mediates collateral formation in zebrafish embryos. Physiol. Genomics 38, 319-327 (2009).

50. Olesnicky Killian, E. C., Birkholz, D. A. \& Artinger, K. B. A role for chemokine signaling in neural crest cell migration and craniofacial development. Dev. Biol. 333, 161-172 (2009)

51. Petit, I., Jin, D. \& Rafii, S. The SDF-1-CXCR4 signaling pathway: a molecular hub modulating neo-angiogenesis. Trends Immunol. 28, 299-307 (2007).

52. Wragg, A. et al. VEGFR1/CXCR4-positive progenitor cells modulate local inflammation and augment tissue perfusion by a SDF-1-dependent mechanism. J. Mol. Med. (Berl) 86, 1221-1232 (2008) 
53. Kidoya, H., Naito, H. \& Takakura, N. Apelin induces enlarged and nonleaky blood vessels for functional recovery from ischemia. Blood 115, 3166-3174 (2010).

54. Yashiro, K., Shiratori, H. \& Hamada, H. Haemodynamics determined by a genetic programme govern asymmetric development of the aortic arch. Nature 450, 285-288 (2007).

55. Lucitti, J. L. et al. Vascular remodeling of the mouse yolk sac requires hemodynamic force. Development 134, 3317-3326 (2007).

56. Buschmann, I. et al. Pulsatile shear and Gja5 modulate arterial identity and remodeling events during flow-driven arteriogenesis. Development 137, 2187-2196 (2010).

57. Nicoli, S. et al. MicroRNA-mediated integration of haemodynamics and Vegf signalling during angiogenesis. Nature 464, 1196-1200 (2010).

58. Yan, C., Takahashi, M., Okuda, M., Lee, J. D. \& Berk, B. C. Fluid shear stress stimulates big mitogen-activated protein kinase 1 (BMK1) activity in endothelial cells. Dependence on tyrosine kinases and intracellular calcium. J. Biol. Chem. 274, 143-150 (1999).

59. Takahashi, M. \& Berk, B. C. Mitogen-activated protein kinase (ERK1/2) activation by shear stress and adhesion in endothelial cells. Essential role for a herbimycin-sensitive kinase. J. Clin. Invest. 98, 2623-2631 (1996).

60. Dimmeler, S., Assmus, B., Hermann, C., Haendeler, J. \& Zeiher, A. M. Fluid shear stress stimulates phosphorylation of Akt in human endothelial cells: involvement in suppression of apoptosis. Circ. Res. 83, 334-341 (1998).

61. Huddleson, J. P., Ahmad, N., Srinivasan, S. \& Lingrel, J. B. Induction of KLF2 by fluid shear stress requires a novel promoter element activated by a phosphatidylinositol 3-kinase-dependent chromatin-remodeling pathway. J. Biol. Chem. 280, 23371-23379 (2005).

62. Clark, P. R. et al. MEK5 is activated by shear stress, activates ERK5 and induces KLF4 to modulate TNF responses in human dermal microvascular endothelial cells. Microcirculation 18, 102-117 (2011).

63. Shen, K. et al. MiR-139 inhibits invasion and metastasis of colorectal cancer by targeting the type I insulin-like growth factor receptor. Biochem. Pharmacol. 84, 320-330 (2012)

64. Wong, C. C. et al. The microRNA miR-139 suppresses metastasis and progression of hepatocellular carcinoma by down-regulating Rho-kinase 2 . Gastroenterology 140, 322-331 (2011).

65. Charo, D. N. et al. Endogenous regulation of cardiovascular function by apelin-APJ. Am. J. Physiol. Heart Circ. Physiol. 297, H1904-H1913 (2009).

66. Gombash Lampe, S. E., Kaspar, B. K. \& Foust, K. D. Intravenous injections in neonatal mice. J. Vis. Exp. e52037 (2014).

67. Helker, C. S. The hormonal peptide Elabela guides angioblasts to the midline during vasculogenesis. eLife 4, e06729 (2015).

\section{Acknowledgements}

We thank M.A. Schwartz for guidance with the flow experiments and critical reading of the manuscript, W. Herzog, C. Helker and A. Schürmann for providing the in situ hybridization probes for the zebrafish apelin receptors, J. Fang for assistance with flow cytometry, A. Dubrac for guidance with retinal analysis, F. Schmalbein for technical assistance, D. Defilippo for administrative assistance, and R. Webber, N. Copeland and W. Evangelisti for assistance with mouse colonies. We thank R. Adams for the Cdh5-CreERT2 mice and T. Quertermous for the Aplnrfl/fl mice,. This study was supported by funding from the US National Institutes of Health (HL095654, HL113005 and HL101284 to H.J.C. and HL75092 to M.A. Schwartz), Howard Hughes Medical Institute (Physician Scientist Early Career Award to H.J.C.), American Heart Association (Established Investigator Award to H.J.C.), National Research Foundation of Korea Grant funded by the Korean Government (NRF-2014S1A2A2028520 to J.K. and H.J.C.), Deutsche Forschungsgemeinschaft (DFG SI-1374/3-1 to A.F.S.), Cells-in-Motion Cluster of Excellence (EXC 1003-CIM to A.F.S.), Sonderforschungsbereich (SFB) 629 and an ERC starting grant (260794-ZebrafishAngio to A.F.S.) and a JSPS Postdoctoral Fellowship for Research Abroad (K.T.).

\section{Author contributions}

I.P., J.K. and H.J.C. conceived the study. I.P., J.K., I.M., S.P., A.L., Y.K., K.T., H.J., Y.K., A.F.S. and H.J.C. performed the experiments. O.F.K. and D.G.A. developed the 7C1 particles. K.R.H generated mouse lines. I.P., J.K., A.F.S., and H.J.C. analysed the data. I.P. and H.J.C. wrote the manuscript.

\section{Additional information}

Supplementary Information accompanies this paper at http://www.nature.com/ naturecommunications

Competing financial interests: The authors declare no competing financial interests.

Reprints and permission information is available online at http://npg.nature.com/ reprintsandpermissions/

How to cite this article: Papangeli, I. et al. MicroRNA 139-5p coordinates APLNR-CXCR4 crosstalk during vascular maturation. Nat. Commun. 7:11268 doi: $10.1038 /$ ncomms11268 (2016)

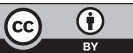

This work is licensed under a Creative Commons Attribution 4.0 International License. The images or other third party material in this article are included in the article's Creative Commons license, unless indicated otherwise in the credit line; if the material is not included under the Creative Commons license, users will need to obtain permission from the license holder to reproduce the material. To view a copy of this license, visit http://creativecommons.org/licenses/by/4.0/ 